\title{
CORRECTION
}

Open Access

\section{Correction to: Aerobic but not Resistance Exercise Can Induce Inflammatory Pathways via Toll-Like 2 and 4: a Systematic Review}

P. A. M. Cavalcante ${ }^{1,2,3,4^{*}}$, M. F. Gregnani ${ }^{5,6,7}$, J. S. Henrique ${ }^{8,9}$, F. H. Ornellas $6,7,10$ and R. C. Araújo $5,6,7,10$

\section{Erratum}

The original article [1] mistakenly omits a grant acknowledgement; thus, the authors would like to acknowledge that the original article was supported by FAPESP 2015/20082-7.

\begin{abstract}
Author details
${ }^{1}$ Medicine (Nephrology) Program, Federal University of São Paulo (UNIFESP), São Paulo, SP, Brazil. ${ }^{2}$ Laboratory of Exercise Genetics and Metabolism, Federal University of São Paulo (UNIFESP), São Paulo, SP, Brazil. ${ }^{3}$ Department of Biophysics, Federal University of São Paulo (UNIFESP), São Paulo, SP, Brazil. ${ }^{4}$ Rua Pedro de Toledo, 669/9and., 04039-032, São Paulo, SP, Brazil. ${ }^{5}$ Molecular Biology Program, Federal University of São Paulo (UNIFESP), São Paulo, SP, Brazil. 'Laboratory of Exercise Genetics and Metabolism, Federal University of São Paulo (UNIFESP), São Paulo, SP, Brazil. 'Department of Biophysics, Federal University of São Paulo (UNIFESP), São Paulo, SP, Brazil. ${ }^{8}$ Neurology/ Neuroscience Program, Federal University of São Paulo (UNIFESP), São Paulo, SP, Brazil. ${ }^{9}$ Exercise Neurophysiology Laboratory, Federal University of São Paulo (UNIFESP), São Paulo, SP, Brazil. ${ }^{10}$ Medicine (Nephrology) Program, Federal University of São Paulo (UNIFESP), São Paulo, SP, Brazil.
\end{abstract}

Received: 9 January 2018 Accepted: 18 January 2018

Published online: 31 January 2018

\section{Reference}

1. Cavalcante PAM, et al. Aerobic but not Resistance Exercise Can Induce Inflammatory Pathways via Toll-Like 2 and 4: a Systematic Review. Sports Med Open. 2017;3:42.

\footnotetext{
* Correspondence: paulaacavalcante@gmail.com

${ }^{1}$ Medicine (Nephrology) Program, Federal University of São Paulo (UNIFESP)، São Paulo, SP, Brazil

${ }^{2}$ Laboratory of Exercise Genetics and Metabolism, Federal University of São Paulo (UNIFESP), São Paulo, SP, Brazil
} 